\title{
STUDIES ON THE PHYSIOLOGY OF THE LIVER
}

\author{
III. THE EFFECT OF ADMINISTRATION OF GLUCOSE IN THE
}

CONDITTON FOLLOWING TOTAL EXTIRPATION OF TIIE LiVER *

FRANK C. MANN, M.D., and THOMAS BIRD MAGATH, M.D.

ROCHESTER, MINN.

In previous studies on the physiolegy of the liver, ' a method of total extirpation of that organ and the effect of the removal on the sugar in the blood were recorded. Since, following removal of the liver the decrease in the blood sugar coincided so exactly with the development of the characteristic symptoms associated with the moribund condition, it was deemed highly possible that a causal relationship existed between the two. An investigation was made, therefore, of the effect of the administration of glucose on animals in which the liver had been removed.

In order to obtain the normal blood sugar value, a specimen of blood was taken from the jugular vein of a dog in which the first two stages of the operation had been completed. The liver was then removed conmpletely in the manner previously described, and close observations made. In many of the experiments the dog was allowed to develop the characteristic symptoms, and glucose was injected intravenously. In other experiments the glucose was administered either by os, by rectum, by jejunostomy, by continuous intravenous injection, or intraperitoneally immediately after hepatectomy; the general condition of the animal and its blood sugar were noted carefully.

The Effect of the Intravenous Injection of Glucose.- The effect of intravenous injection of glucose on an animal dying from the remoral of the liver is one of the most remarkable physiologic phenomena we have ever observed. The animal, comatose and perfectly flaccid, apparently unable to contract any muscle, except the diaphragm, is restored immediately to a seemingly normal condition by the injection of from 0.25 to $0.5 \mathrm{gm}$. glucose for each kilogram of body weight. We have seen such animals stand thirty seconds after the injection of glucose, walk, respond to call, wag their tails, drink water, and so forth, in less than one minute from the time they had been perfectly flaccid. If the animal is allowed to develop the convulsions, which are quickly followed by death when untreated, the results are even

* From the Division of Experimental Surgery and Pathology, The Mayo Foundation, and the Section on Clinical Laboratories, Mayo Clinic.

1. Mann, F. C.: Studies on the Physiology of the Liver. I. Technic and General Effects of Removal, Am. J. M. Sc. 161:37 (Jan.) 1921. Mann, F. C., and Magath, T. B.: The Effect of Total Removal of the Liver, Tr. Sect. Path. \& Physiol., A. M. A., 1921, p. 29. 
more striking. The convulsions stop immediately, consciousness returns, and in a minute or two the animal walks around normally. Restoration to normal is usually possible by the injection of glucose at any stage of the moribund condition until the heart has actually stopped. We have restored an animal after the cessation of respiration by maintaining artificial respiration until the glucose could be injected.

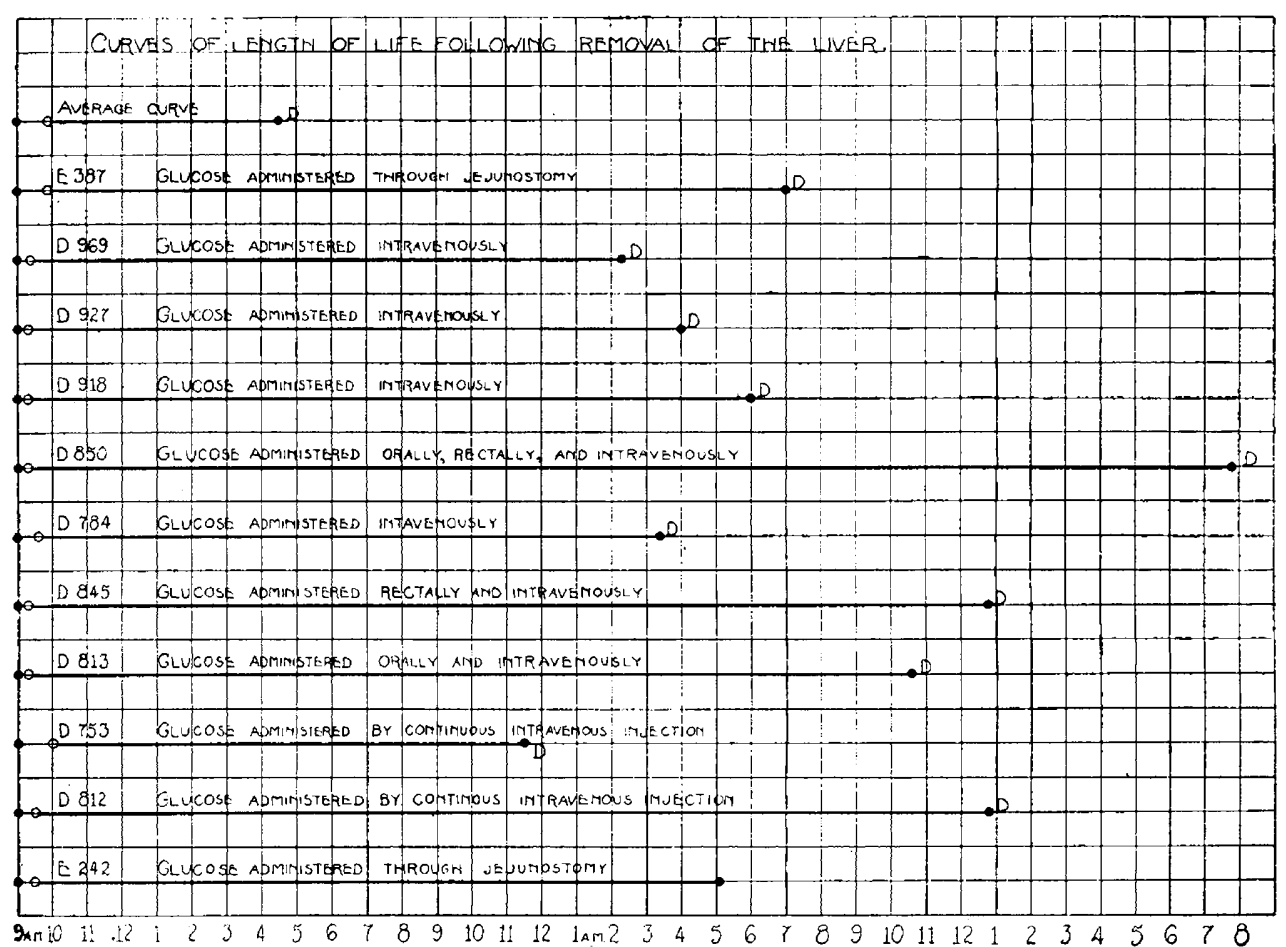

Fig. 1.-O: Time of removal of liver. D: Time of death. The first curve represents the average length of life in the average untreated hepatectomized animal. The curve was constructed and the averages obtained from six animals dying from results of uncomplicated hepatectomy. The second curve represents the length of life following removal of the livers of various animals after the administration of glucose. Note the great increase in length of life due to the glucose administered.

Immediately after the injection of glucose, the blood sugar reaches a very high level; at first it decreases quickly, then falls more slowly. As the level again becomes low, the typical symptoms reappear. The time this occurs after injection depends on many factors. Some of these are: the amount of glucose injected, whether the animal is active or quiet, and whether or not it is kept warm. Restoration of the animal from the comatose condition can be repeated many times by the 


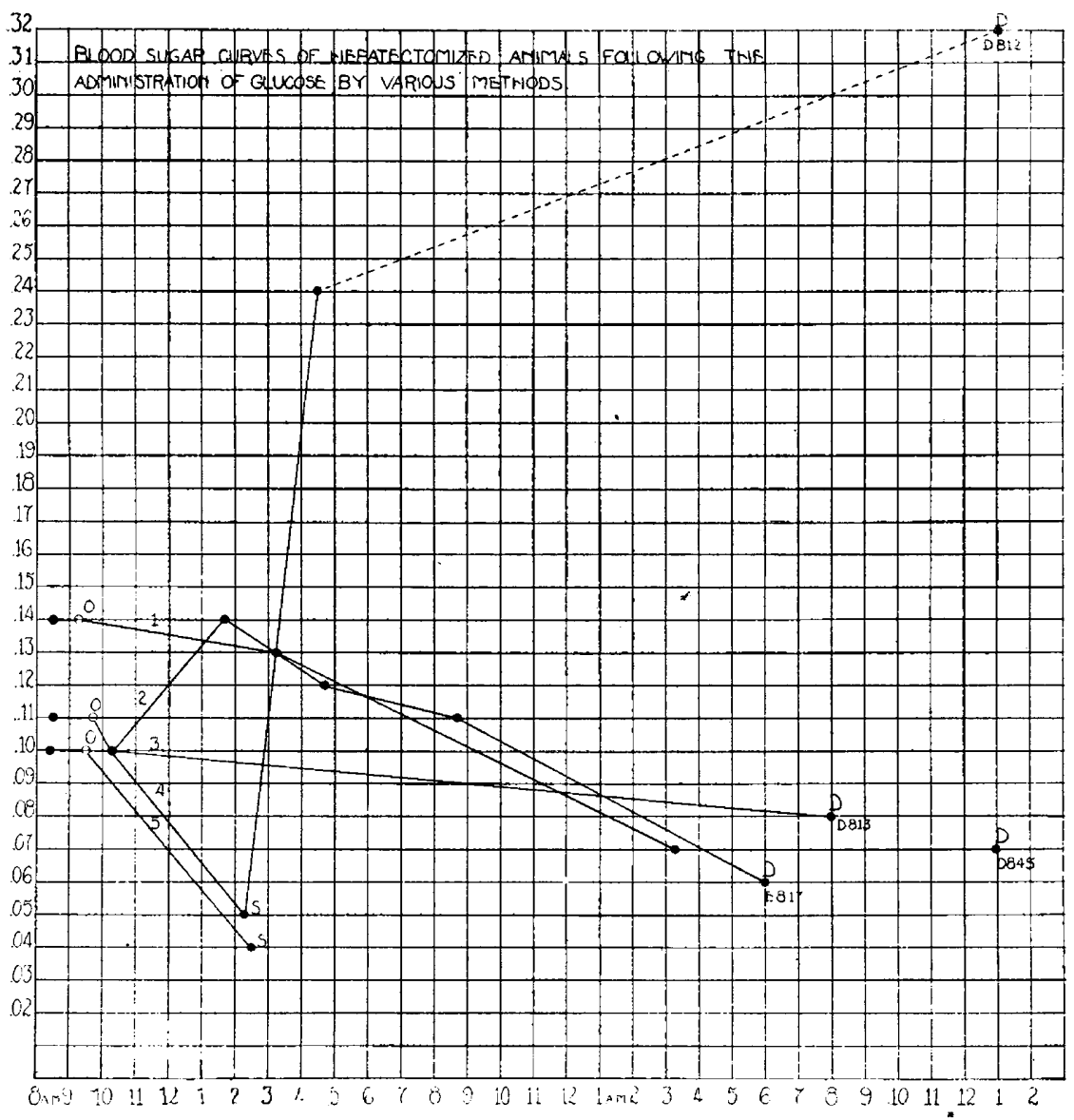

Fig. 2.-O: Point of removal of liver. S and D respectively: Development of symptoms and death. The first curve represents the blood sugar after the administration of glucose by rectum for many hours. Absorption was not sufficient to maintain correct blood sugar level and glucose also was given intravenously for the last eight hours of the experiment. The second curve represents the blood sugar after the administration of glucose through a jejunostomy, and the blood sugar level was maintained. The third curve represents the blood sugar level during the administration of glucose orally and the blood sugar level was maintained throughout the experiment. The fourth curve represents the blood sugar without the administration of glucose until the animal had developed the first symptoms when it was given intravenously. The fifth curve illustrates the average course of blood sugar following removal of the liver. Note the increase in the length of life when the blood sugar level was maintained at normal or above normal. 
injection of glucose. In each instance, its clinical condition exactly coincides with the blood sugar level. Finally, however, usually after many restorations, it may be noted that the same amount of glucose does not maintain the animal in a normal condition for so long a time and the characteristic symptoms develop at a higher blood sugar level.

The Character of the Symptoms that Develop with a High Blood Sugar Level After the Administration of Glucose.-At this stage of the experiment another condition develops and other symptoms appear. These differ with different animals. In some experiments the animal suddenly becomes comatose and remains so for a variable period, usually not more than an hour, and dies suddenly and quietly. In

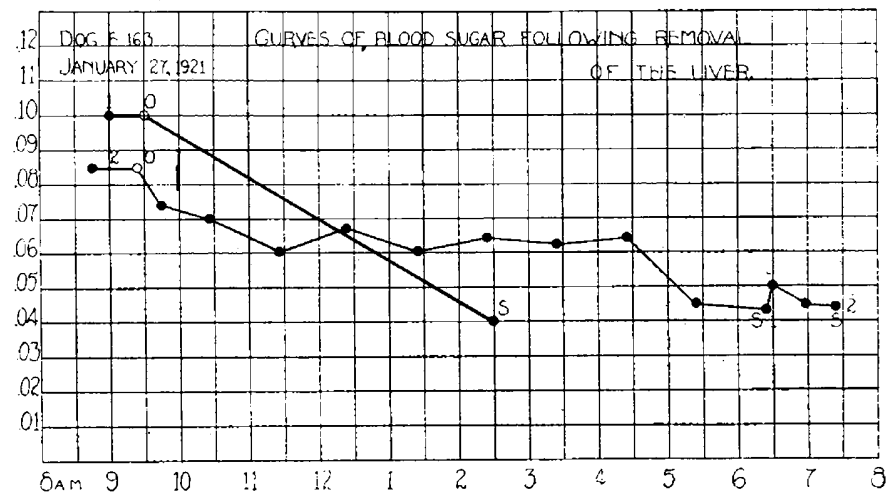

Fig. 3.-The first curve shows the average change in blood sugar following removal of the liver. The second curve shows the gradual decrease in blood sugar of a dog kept very quiet after operation and artificial heat applied. $\mathrm{O}$ : Liver removed. $\mathrm{S}_{1}$ : Animal moribund; $0.125 \mathrm{gm}$. glucose for each kilogram of body weight was injected. This barely increased the blood sugar but restored the animal so that it could walk. This is the minimal amount of glucose which will produce a reaction when the animal is moribund.

other experiments the symptoms are similar to those following excessive feeding of meat to animals with Eck fistulas. The animal is at first quite restless, ataxia then appears, and sight and hearing are lost. Coma develops and death is sudden and quiet. Anuria ustally accompanies this second moribund condition; the blood sugar level may be high and the injection of glucose is without beneficial effect.

The clinical appearance of the condition associated with low blood sugar and that with high blood sugar, is quite different. There is no difficulty in noting when the second condition is developing, but the rate at which it develops cannot be anticipated as it can in the first condition which is associated with a decrease in blood sugar. The cause of the second condition has not been determined, but it seems to be dependent on some change in metabolism other than carbohydrate. 
The Effect of Administration of Glucose by Continuous Intravenous Injection, Through the Alimentary Tract and Intraperitoneally. -In some experiments, before the blood sugar had decreased to a low level and before symptoms occurred, glucose was injected slowly and at a fairly uniform rate with a continuous injection machine. In this manner the blood sugar level was kept normal or slightly above normal. The first group of symptoms never developed in these animals, but after from eighteen to twenty-four hours, the second group developed and the animals died.

In one series of experiments, an attempt was made to maintain the normal blood sugar level by the injection of glucose into the rectum.

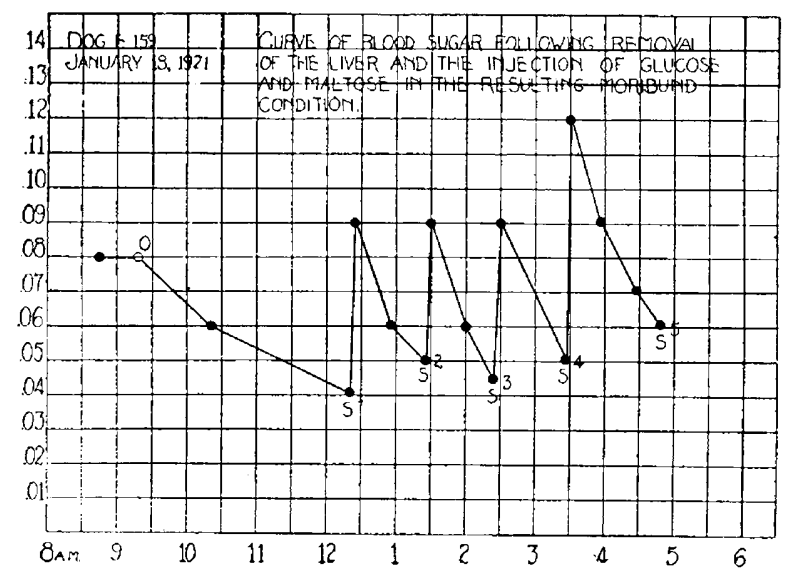

Fig. 4.--O: Liver removed. Blood sugar which was rather low before operation decreased immediately and three hours after it was only $0.04 . \mathrm{S}_{1}$ : The animal was moribund; it was restored to normal by the injection of $0.25 \mathrm{gm}$. glucose for each kilogram of body weight. $S_{2}$ : After one hour when it was necessary to repeat the injection. $S_{3}$ : Injection again repeated. $S_{4}$ : At the end of the next hour symptoms developed and $0.25 \mathrm{gm}$. maltose for each kilogram of body weight was injected. The maltose restored the animal in exactly the same manner as the glucose, except that the period of recovery was slower. Note the uniform rise in blood sugar following glucose injection, and that $0.25 \mathrm{gm}$. for each kilogram of body weight appeared to last one hour.

In this we were only partially successful, because in many instances a considerable amount of injected fluid was rejected. However, the blood sugar level remained higher and the first group of symptoms appeared later than in animals not given glucose. In some cases enough glucose was absorbed so that the first group of symptoms did not develop but the animals died in from eighteen to twenty-four hours following the development of the second group of symptoms.

In some experiments the glucose was given by mouth, a few cubic centimeters of a weak solution, for example 10 per cent. administered every few minutes beginning as soon as the animal recovered from 
the immediate effects of the operation. These experiments were very successful so far as keeping the animal alive was concerned. In most cases the blood sugar was maintained at a normal level and the animal never developed the first group of symptoms, but died after the appearance of the second group. By a combination of oral and intravenous administration we were able to keep an animal alive thirty-four hours after complete removal of the liver.

The chief difficulty encountered with the oral administration was that the operative trauma sometimes interfered with the emptying of the stomach so that most of the glucose did not pass into the intestine where it could be absorbed. To obviate this a jejunostomy was made in some experiments at the time the liver was removed. The administration of glucose through a jejunostomy tube was the most satisfactory method used. We were able also to keep the blood sugar level normal by the intraperitoneal injection of glucose.

The Constancy of the Effect of Glucose.-We observed the resuscitating action of glucose administered intravenously, more than 200 times in about forty hepatectomized animals. It never failed to restore to normal an animal that had developed the group of symptoms associated with low blood sugar. Further we administered glucose to twenty-five hepatectomized animals by the other methods mentioned, and in none in which we were successful in maintaining a normal blood sugar value or a hyperglycemia, did the first characteristic group of symptoms develop.

The Effect of Other Substances Administered.- We attempted to determine whether the restorative action of glucose in the moribund condition following hepatectomy was specific. For this purpose a wide variety of substances which would seem to give some promise of having a related action, were tested in the same manner and in amounts which seemed to correspond to the amount of glucose necessary to restore the hepatectomized animal to normal. The following substances tested were found not to have a restorative action:

Saccharose; lactose; levulose; inulin; sodium chlorid; sodium sulphate; sodium carbonate; sodium bicarbonate; ethyl alcohol; glycerol ; lactic acid (racemic); acetic acid; hydrochloric acid; pyruvic acid; epinephrin; pituitary extract; glycocoll.

Only four substances were found, besides glucose, which had a beneficial action when injected into the moribund animal with a low blood sugar following removal of the liver. These are maltose, mannose, dextrin and galactose.

The action of maltose is somewhat cumulative. When it is first injected in an animal with the characteristic symptoms following hepatectomy, the recovery is very slow but complete. Ten minutes may elapse between the time of injection and the time the animal 
can walk, whereas when glucose is injected recovery is usually complete in from one to four minutes. When the animal becomes moribund again and maltose is again injected, the recovery is much faster than after the first injection. Mannose produces a response similar to maltose. Dextrin produces a definite but very slight beneficial action. The animal will probably regain consciousness and muscie tone but

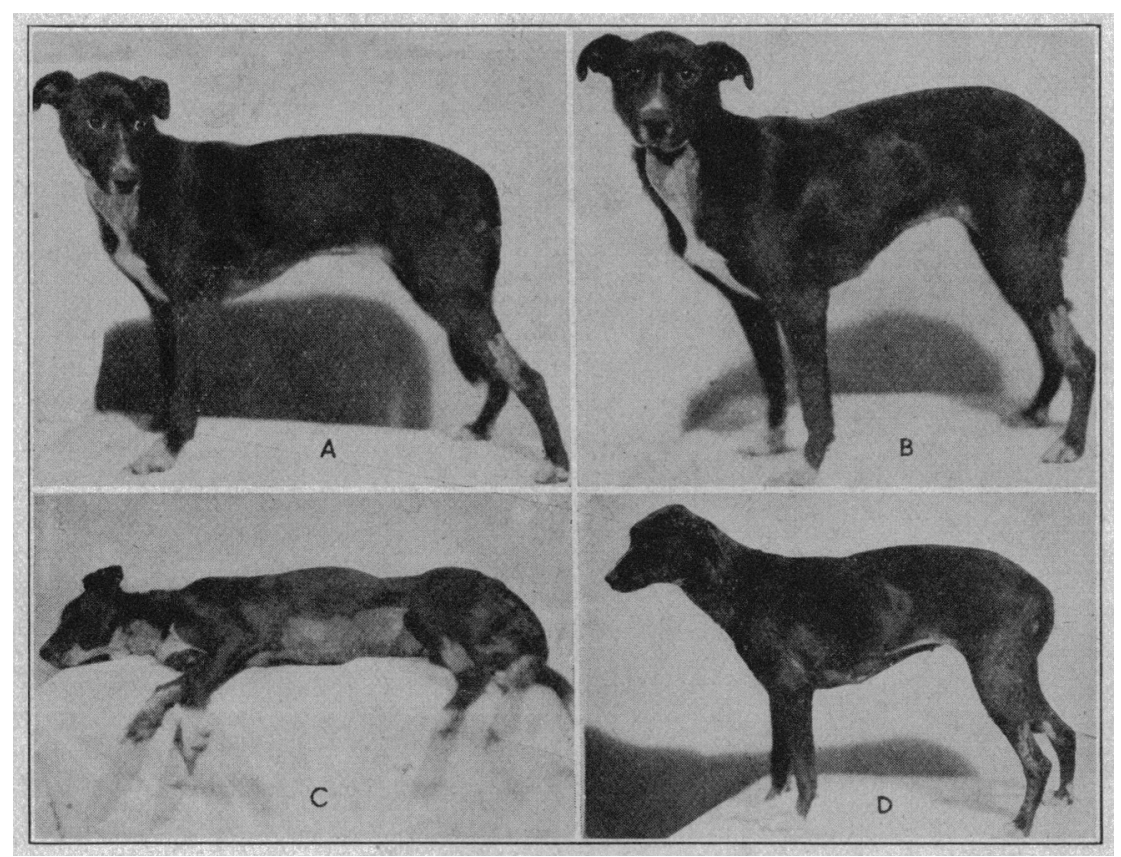

Fig. 5.-A: Animal just preceding total removal of the liver. Two previous operations have been performed: (1) A reverse Eck fistula; (2) ligation of the portal vein. Blood sugar at this time, 0.10 per cent.

$B$. Animal one hour after removal of the liver and less than two hours after the photograph shown in A. Note that the animal appears normal.

C. Animal moribund, six and one-half hours following the total removal of the liver. The animal flaccid, with a few muscular twitchings. Without treatment this animal would probably have been dead within another hour. Blood sugar at this time, 0.05 per cent.

D. Animal four minutes after the injection of glucose and five minutes after the picture shown in $C$. The animal again appears normal.

will not be able to stand when dextrin is injected. The beneficial action of galactose is so slight and transient that it would scarcely be noted unless the animal were under the strictest observation. While we have not determined why certain substances are beneficial in the restoration of an hepatectomized animal to normal it should be noted that maltose is supposed to be converted into glucose in the blood stream. If this is true, it explains the beneficial effect of maltose on hepatec- 

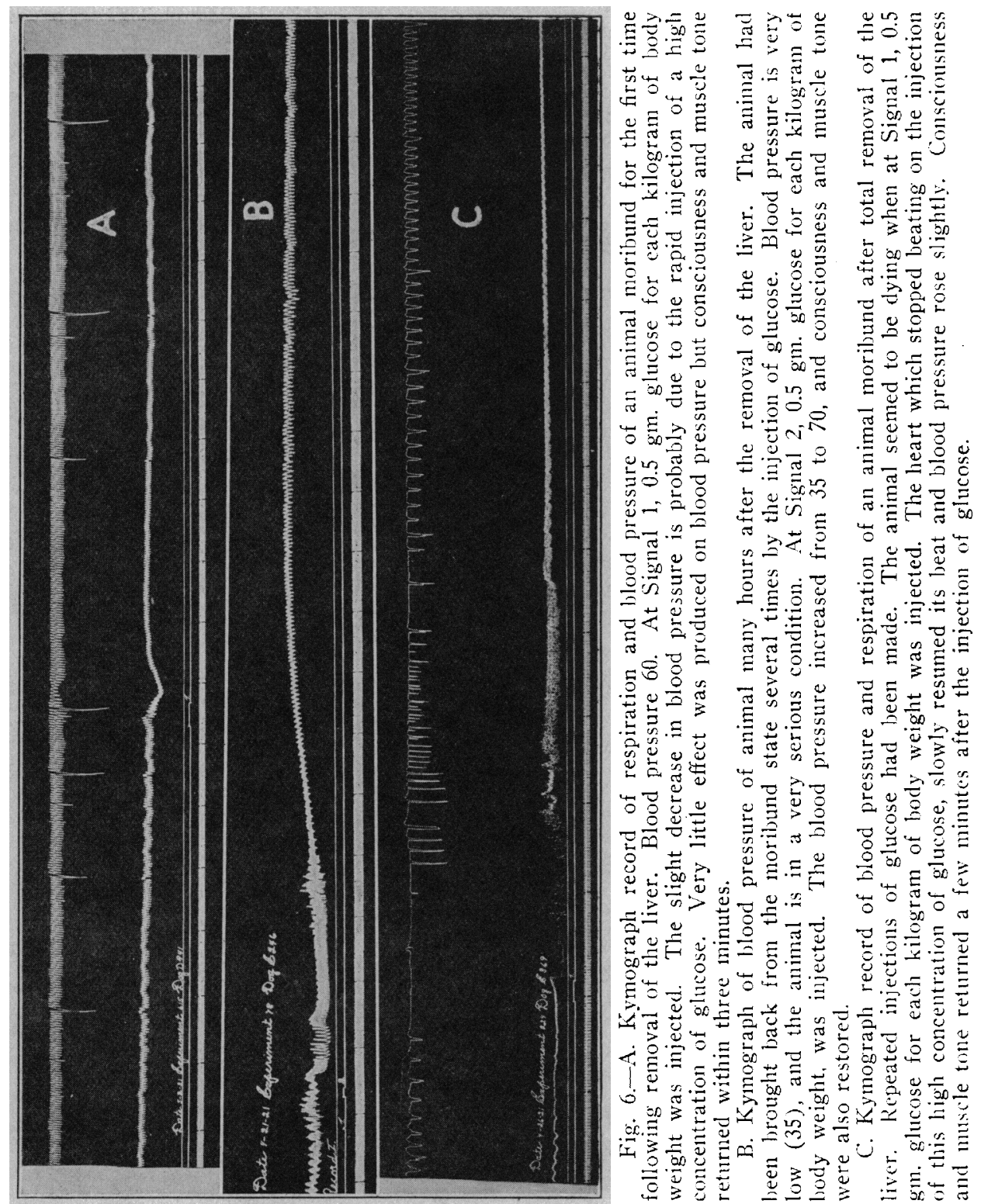
tomized dogs, and the release of excess maltose in the blood after the first injection explains the better and more prompt effect of the second. We suggest that mannose also may be changed into glucose in the blood stream. The beneficial effect of dextrin may be explained on the basis that it contains maltose and glucose as impurities.

The Amount of Glucose Necessary to Restore the Animal to Normal.-While there has been individual variation in the reaction of the hepatectomized animal to various amounts of glucose, in general the quantitative relationship has been constant. The smallest amount of glucose which will produce a definite beneficial action in the moribund hepatectomized $\operatorname{dog}$ is $0.125 \mathrm{gm}$. for each kilogram of body weight. Smaller amounts than this usually do not produce noticeable effects, or produce questionable effects. One-eighth gram of glucose for each kilogram of body weight restores the animal to consciousness and enables it to stand, but not to regain its normal strength, and in a short time, from fifteen to thirty minutes, the comatose condition returns. When $0.25 \mathrm{gm}$. of glucose for each kilogram of body weight is injected, the recovery is prompt and complete. In our routine work we found that $0.5 \mathrm{gm}$. of glucose for each kilogram of body weight was the most satisfactory amount.

The Amount of Glucose Necessary to Maintain the Animal in a Normal Condition.- The amount of glucose to keep the hepatectomized animal normal is subject also to individual variation, but on the whole is constant. In the average animal, kept quiet in a warm room, 0.25 gm. glucose for each kilogram of body weight maintains it for one hour. If the animal is flaccid and $0.25 \mathrm{gm}$. for each kilogram of body weight is injected intravenously, the animal will be restored to normal and will not need another injection for about an hour. Our routine injection of $0.5 \mathrm{gm}$. glucose for each kilogram of body weight usually was sufficient to maintain the animal for two hours. The effect of various experimental procedures, activity, and cold, often make it necessary to repeat the injections more frequently.

The Gencral Effects of the Glucose Injection.-The symptoms associated with the decreasing blood sugar following hepatectomy seem to show that at first there is a depression and later a stimulation of the central nervous system. We have not as yet determined definitely the nature of this process or the component parts of the nervous system acted on. In any event the injection of glucose abolishes completely all symptoms referable to the nervous system.

The effect of glucose injection on blood pressure depends on the condition of the latter at the time of injection. If the pressure is almost normal, as it is at the beginning of an experiment, the glucose causes but slight effect. If the pressure is low as occurs after an animal has been restored many times, the glucose causes a distinct rise in pressure. 
The heart rate, which is usually very rapid after hepatectomy, may become slower when the blood sugar is very low. When the animal has developed well marked symptoms, it is usually irregular. The injection of glucose, regardless of the rate of heart beat produces a beneficial action, making it stronger and more regular. Electrocardiographic tracings have shown no change either when the animal was flaccid or after recovery following glucose injection.

In many of the experiments the irregularity of the respiration is marked. Following the glucose injection the respiratory movements return to normal.

The general temperature curve is downward throughout the course of an experiment. In some instances when this factor was observed closely, it rose slightly following glucose injection. In most experiments, the more rapid decrease in temperature occurred while the animal was moribund.

\section{DISCUSSION}

The explanation of the decrease in blood sugar following hepatectomy and of the remarkable restorative action of glucose when the animal seemed to be dying, is probably the same. ${ }^{2}$ The chief possibilities in regard to this explanation were discussed in a previous paper. ${ }^{1}$ Our further investigations enable us to elaborate on this discussion.

In view of the current idea that the liver is of great importance in regulating the reaction of the blood it was conceived that the removal of the organ might produce a condition either of alkalosis or acidosis, and that the glucose acted in some way to restore the hydrogen ion content of the blood. However, we have never found sufficient change in the hydrogen ion concentration or the carbon dioxid combining power of the blood at the time the first group of symptoms developed to warrant such a consideration. Furthermore, the injection of both acids and alkalis make the condition worse.

The action of glucose may also be explained by its osmotic power of changing the fluid concentration of the body cells and the composition and volume of the blood, thus washing out and diluting toxic substances which developed in the cells because of the absence of the liver. That this is not the manner by which glucose produces the remarkable effect is proved amply by the fact that other substances having even greater osmotic strength, such as hypertonic solutions of sodium sulphate and sodium chlorid, do not produce beneficial action when injected in large amounts. The injection of other sugars such as levulose, saccharose, and lactose is of no value, while a weak

2. Mann, F. C., and Magath, T. B.: Studies on the Physiology of the Liver. II. Effect of Removal of the Liver on the Blood Sugar Level, Arch. Int Med. 30:73 (July) 1922 . 
solution of glucose restores to normal. A simpler explanation and one which agrees with most of our definitely proved facts, is as follows:

When the liver is removed a considerable proportion of the glucose storage capacity and the mechanism for elaboration of glucose is destroyed. The tissues use the sugar in the blood which for a variable period is partially maintained by the transformation into glucose of the available glycogen in the muscles. As the tissues utilize the glucose faster than it can be liberated from the muscles, blood sugar decreases as soon as the liver is removed. When the blood sugar has decreased below a certain amount, the concentration in the cells of the body becomes too low to maintain the normal cellular metabolism. This leads to the development of the characteristic symptoms described. The injection of glucose furnishes another food supply and cellular metabolism returns. While this is the most simple explanation of our facts, the final explanation will depend on the correlation with this of many other factors, for example the suddenness of the decrease in blood sugar.

The central nervous system seems to be early and markedly affected by the decrease in blood sugar. While the entire phenomenon which we have been studying can be referable to an effect on the central nervous system, at present it seems best to consider it a general bodily reaction.

\section{SUMMARY}

The total removal of the liver is followed by a definite and progressive decrease in blood sugar. Coordinate with this decreasing blood sugar, a characteristic group of symptoms develops. If glucose in suitable amounts is injected intravenously into an animal exhibiting these characteristic symptoms, it is immediately restored to normal. If glucose is administered so that the blood sugar does not decrease, the symptoms do not develop. 